\title{
Conference Scene
}

Summary report from EAACl: London 2010

\section{9th Congress of the European Academy of Allergy and Clinical Immunology 5-9 June 2010, London, UK}

Immunotherapy is, to date, the only effective curative method for the treatment of allergic disorders. Great efforts have been made to improve the efficacy, safety and patient compliance with this method. The growing understanding of the immunological mechanisms underlying immunotherapy has led to new approaches for immunotherapy involving the routes of administration and the kinds of molecules used. In addition, new vaccines are being created that combine the advantageous immunological charasteristics of different substances, such as virus-like particles linked to allergens. Many new results from ongoing research into these topics were presented at the 29th congress of the European Academy of Allergy and Clinical Immunology in London.

European scientists and doctors specializing in allergology and clinical immunology met for the 29th Congress of the European Academy of Allergy and Clinical Immunology in London, UK, 5-9 June 2010. A central topic for this community is improvement and new developments in specific immunotherapy (SIT), a treatment that stimulates immunological tolerance of allergens responsible for allergic responses in the context of atopic dermatitis, urticaria, food allergies, allergic rhinoconjunctivitis and allergic bronchial asthma. Next year will be the 100th anniversary of SIT, the concept of which was first published 99 years ago in Lancet by Noon et al. [1]. Ever since, scientists and doctors have been working on improving pharmacological standardization and optimizing the allergen extracts and routes of administration so as to improve clinical efficacy, develop adjuvants that strengthen the immune response and ensure the identification of patients who will profit the most from each method of therapy. To date, the mechanisms of SIT are still not fully understood, leading to a growing body of experimental work investigating immunological processes during immunotherapy.

This year, a number of new studies and approaches in SIT were presented in London. Great efforts have been made to improve the efficacy and safety of SIT in the past few years, particularly since the latter is the limiting factor of this treatment. In very rare cases, SIT administered via subcutaneous injection (SCIT) can lead to systemic reactions and occasionally to anaphylactic shock. Therefore, not only is the investigation of new routes for the application of SIT warranted, but also the development of new vaccines that are more effective and cause less side effects. Furthermore, new insights into the immunological mechanisms underlying a successful SIT were described at the meeting.

As an alternative to SCIT, the sublingual route of administration (SLIT) has become of great interest during the past few years, especially because the development of allergen tablets has significantly improved its pharmacokinetics and pharmacodynamics via better resorption. A number of high-quality clinical studies investigating the efficacy and safety of this method have been carried out. In particular, the long-term effects of allergen immunotherapy tablets (AIT) were the focus in these studies. Three different AITs were presented:

- Using Oralair ${ }^{\mathrm{TM}}$ (Stallergenes, Antony, France) tablets, a reduction of symptom scores (mean: 35\%; median: 50\%) over a period of 3 years was observed. During this period, a booster effect in the success of therapy was observed. As a great advantage of this therapy, only mild local side effects and no severe systemic reactions were observed, which led to the conclusion that therapy with OralAir is efficient and safe;

- For Grazax ${ }^{\circledR}$ (ALK-Abelló, Hørsholm, Denmark), 5-year data (2 years without treatment) were presented by Stephen Durham (Imperial College, UK). The standardized-quality immunotherapy tablet was demonstrated to induce a diseasemodifying and continuous long-term

\section{Matthias Stiehm ${ }^{1}$ \& Albrecht Bufe ${ }^{\dagger 1}$}

${ }^{1}$ Ruhr-University Bochum, Department of Experimental Pneumology, Bürkle-de-la-Camp-Platz 1,

44789 Bochum, Germany

${ }^{+}$Author for correspondence:

Tel.: +492343024510

Fax: +492343024682

albrecht.bufe@rub.de

\section{Financial \& competing interests disclosure}

The authors have no relevant affiliations or financial involvement with any organization or entity with a financial interest in or financial conflict with the subject matter or materials discussed in the manuscript. This includes employment, consultancies, honoraria, stock ownership or options, expert testimony, grants or patents received or pending, or royalties.

No writing assistance was utilized in the production of this manuscript. 
effect in adults. The tablet was investigated in a randomized, double-blind, placebo-controlled Phase III trial. Levels of the $\mathrm{IgG}_{4}$ - and IgE-blocking factors remained elevated even 2 years after finishing 3 years treatment. The active group showed a $31 \%$ reduction in median rhinoconjunctivitis symptom score;

- The Bet v1 SLIT (Stallergene) also resulted in an increase in the level of $\mathrm{IgG}_{4}$-blocking antibodies. Interestingly, no dose-dependency of effects could be observed in this study, probably owing to the fact that the applied allergen concentrations were already in the saturation phase. However, the highest incidence of side effects was found in the group treated with $50 \mu \mathrm{g}$ allergen.

Albrecht Bufe (Ruhr-University Bochum, Germany) discussed the growing importance of SLIT as a therapeutic method for children. He pointed out that side effects and poor compliance limit the use of SIT in children, and that the development of allergen tablets might provide a better route of treatment. Although long-term data on AITs are available, evidence for the efficacy of SLIT in children is still lacking. Thus, only SLIT products whose efficacy has been proven are recommended for clinical use. Since SLIT is a home-based treatment, it is very important to document risk factors and inform patients and parents about the expected course of these risks in order to improve compliance and improve the voluntary entry of patients into the ambulance.

In addition to SCIT and SLIT, a third method has become of interest during the past few years, namely intralymphatic immunotherapy. The allergen is directly injected into the lymph nodes, and the method has proven to be a promising alternative to SCIT and SLIT [2,3]. It was demonstrated in several studies that immune responses to protein, peptide and naked DNA vaccines are enhanced using intralymphatic immunotherapy, leading to a reduced number of allergen doses needed and a reduced allergen concentration. Although highly dependent on the technical skills of the doctor, as a painless method, intralymphatic immunotherapy also improves convenience and patient compliance.
In order to achieve a highly effective and convenient therapy, both the route of administration of SIT and the allergens themselves must be optimized. An ideal vaccine for desensitization should be highly immunogenic and should alleviate allergic symptoms after a few injections while being nonreactogenic. In particular, virus-like particles (VLPs) from the bacteriophage $\mathrm{Q} \beta$ appear to be a promising alternative to the common allergen extracts, allergoids or recombinant allergens. Thomas Kündig (University Hospital Zürich, Switzerland) explained that the transfer of allergen to the draining lymph nodes can be increased owing to covalent coupling of the mite allergens to VLPs. After the first injection, an IgG response occurs. In a Phase IIa study, after 6 months of treatment, a 100-times increased allergen tolerance in nasal provocation test was observed. VLPs for SIT are also used for other allergens, such as Fel d 1 [4]. The main advantages of using VLPs are a rapid immune response and the avoidance of severe side effects, such as anaphylaxis. Since VLPs are densely packed, B-cell activation without $\mathrm{T}$-cell help is possible. The effects of IgG antibodies are mainly mediated by the intracellular Fc $\gamma$ R $2 b$ pathway. In addition, the degranulation of basophils is inhibited by IgG.

Another central topic concerning novel vaccines in immunotherapy was allergen peptides. Allergen peptides are an effective and interesting alternative to the commonly used vaccines. As presented by Mark Larché (Asthma UK Centre for Allergic Mechanisms of Asthma, UK), the Fel $\mathrm{d} 1$ prototype vaccine (the major allergen of cats) does not lead to mastcell activation, since $\operatorname{IgE}$ antibodies are not crosslinked by the peptides. This is due to a noneffective binding of peptides to $\operatorname{IgE}$ antibodies as shown in vitro [5]. Studies from Larché et al. broaden the understanding of mechanisms of peptideinduced tolerance in allergic asthma and might be important for the design of peptide interventions to ameliorate chronic allergic and autoimmune diseases. The vaccine consists of a combination of different overlapping peptides of Fel $\mathrm{d} 1$. The data indicate that peptide immunotherapy acts via an IL-10-dependent mechanism. In human patients who are allergic to 
the cat allergen, the therapy induces a process involving linked epitope suppression associated with induction of IL-10. Induction of clonal T-cell anergy was not observed. In a murine model, a reduction in the symptoms of allergic asthma could be observed, including reduced Th2 cytokine production, reduced airway eosinophilia and lowered IgE production. An important point was that the percentage of IL-10-producing $\mathrm{T}$ cells in the lung was markedly enhanced after peptide immunotherapy. This percentage was even greater than the percentage of cells specific for the treatment peptide. In the murine model, the induction of tolerance was independent of TGF- $\beta$ and Foxp3. Francois Spertini (Centre Hospitalier Universitaire Vaudois, Switzerland) discussed contiguous overlapping Bet v 1-derived peptides and showed the results from a Phase I/IIa clinical trial in birch pollen-allergic volunteers. The overlapping peptides, with an average length of 50-70 amino acids also do not bind $\operatorname{IgE}$ in vitro. Since no reduction in temperature was observed by treating mice with these peptides, an induction of anaphylactic reactions seems to be mostly excluded. In skin prick test, reactions were a 100-times lower compared with the natural allergen. No immediate reactions were observed after injections, and only local reactions appeared at the injection site. An increase in T-cell proliferation, IFN- $\gamma$, IL-10 and $\mathrm{IgG}_{4}$ levels were observed as a result of the treatments. Rudolf Valenta (Medical University of Vienna, Austria) presented a refined immunotherapy concept, based on the application of peptides derived from allergen surfaces that exhibit reduced, allergen-specific $\mathrm{IgE}$ as well as T-cell reactivity [6]. These peptides enhance the allergen-specific protective IgG response with T-cell help from a nonallergenic carrier molecule. Their data demonstrated that these peptide vaccines are able to bypass allergen-specific $\operatorname{IgE}$ and T-cell activation. Therefore, they can be administered at high doses without IgE- and T-cellmediated side effects. Clinical trials with these vaccines still have to be performed, but it is expected that these drugs should be a safe and convenient form of SIT as an alternative to symptomatic, drug-based allergy treatment.
Another approach to optimize efficacy of SIT was simply to 'teach old protocols new tricks'. It is the ratio between adjuvant and allergen that is important in the improvement of efficacy and safety of SIT, as presented by Kirsten Jung (Erfurt, Germany). Mixture of allergen extracts with aluminium hydroxide leads to a slow release of allergen molecules. It was shown that an alteration in the ratio of adjuvant:allergen, by reducing either the allergen or adjuvant concentration, can lead to reduced side effects and an increase in $\mathrm{IgG}_{4}$ production in SCIT preparations. Furthermore, a reduction of the maximum dose applied for maintenance of therapy and shorter updosing schedules can be achieved. In a clinical trial, the safety and tolerability profile of a product with an optimized allergen:aluminium hydroxide ratio was investigated. It could be shown that a five-injection updosing schedule (each injection 1 week apart) with an optimized allergen:adjuvant ratio was well tolerated and led to less systemic and local side effects compared with a schedule where injections were performed every 3-4 days. However, comparative clinical studies with conventional dosages in terms of clinical efficacy are missing.

Since Noon performed the first immunotherapy in 1911, SIT has been the only successful curative method for hyposensitization. However, the mechanisms are still not fully understood. In particular, mechanisms underlying sublingual immunotherapy came into focus recently. Robyn O'Hehir (Alfred Hospital, Australia) reported in the workshop, "Where are we with SLIT?", that, similar to SCIT, an increase of $\mathrm{IgG}_{4}$-blocking antibodies is also observed in SLIT. Furthermore, a reduced activation of basophils and reduced $\mathrm{CD}^{+}$ T-cell proliferation occur following SLIT. If peripheral blood mononuclear cells are stimulated with house dust mites, a reduced IL-5 production of these cells occurs, and after 2 years, an increase in IL-10 production appears. Two groups of $T$ cells are of importance for this effect. CD $4^{+} 25^{+} \mathrm{CD} 127^{\text {low }}$ (IL-7 receptor) cells, a subgroup of Tregs, suppress the CD4 $4^{+} 25^{+}$ CD127 $7^{\text {high }}$ Treg cells. The efficacy of SLIT is greater during the peak of the season.

Another very interesting observation was presented by Jean-Pierre Allam (University of Bonn, Germany) who showed data on 
human skin and oral mucosal dendritic cells (DCs). It has been suggested that mucosal DCs are able to induce both proinflammatory and tolerogenic immune responses. Allam demonstrated that nasal mucosal DCs strengthen allergic immune responses in atopic individuals, while oral mucosal Langerhans cells induce a regulatory immune response. These cells can support the immunological homeostasis within oral mucosal tissue. They can also induce the desired allergen-specific tolerance during SLIT. It was reported that oral mucosal DCs do not mature to as great a degree by allergen uptake as skin DCs. This phenomenon leads to the conclusion that the maturation status of antigenpresenting cells has a great influence on tolerance induction [7]. Laurent Mascarell (Stallergènes SA, France) also presented data on cells responsible for tolerance induction via the sublingual route. $\mathrm{He}$ demonstrated that macrophage-like cells play a pivotal role in tolerance induction. In his talk, he pointed out that addressing these cells during SLIT should be a future task. The process of designing new vaccines for SLIT should be adjusted to meet this task. The targeting of DCs for the treatment of allergic disorders was also discussed by Thomas Bieber (Friedrich-Wilhelms University, Germany). He explained that Langerhans cells were responsible for tolerance induction. During the treatment of atopic dermatitis, the number of these cells increases in the skin while the number of inflammatory dendritic epidermal cells decreases in inflamed skin.

Nathalia Novak (University of Bonn, Germany) presented data on the early activation of basophils via histamine receptor 2. As a result, memory Tregs were induced, which could secrete IL-10 and TGF- $\beta$ in beekeepers $[8,9]$. During the late response, the ratio between inflammatory and noninflammatory antibodies ( $\left.\operatorname{IgE}: \operatorname{IgG}_{4}\right)$ is of great importance. While $\mathrm{T}$ cells induce $\mathrm{IgG}_{4}$ production, Tregs inhibit IgE-producing cells. The induction of Tregs could be observed owing to upregulation of Foxp $3^{+}$cells in the tonsils. It is known that tonsils can induce Tregs, which themselves are able to induce tolerance [10]. Furthermore, plasmacytoid DCs are able to induce Foxp $3^{+}$Tregs. One of the main questions was whether these Tregs were antigen specific. Since there was an increase from 0.03 to $0.38 \%$ Tregs caused by Bet v 1 tetramere, an antigen-specific induction of tolerance is likely [11].

Moises Calderon (Royal Brompton Hospital, UK) presented data on the important role of anaphylaxis, which is a mostly underestimated and differently described the side effect of immunotherapy. He pointed out the need for an exact documentation and the future creation of questionnaires for the European situation concerning the prevalence of anaphylaxis. It is side effects such as anaphylaxis that limit the use of immunotherapy. However, severe side effects are more commonly observed in SCIT than in SLIT. The role of systemic reactions in SIT was also discussed in an immunotherapy interest group; a Europe-wide survey on the documentation of systemic side effects is planned. A simple strategy for such surveys was presented by Birnbaum. The problem is deciding which classification of systemic side effects should be used. There is a novel and very precise classification published by Cox in 2010 [12], but this classification is complicated in practical use.

Taken together, a number of new trends in immunotherapy were presented at the meeting in London, indicating that several questions remain unanswered. We look forward to listening to further innovative steps next year in Istanbul, Turkey, where the 30th Congress of the European Academy of Allergy and Clinical Immunology 2011 will take place.

\section{Bibliography}

1 Noon L, Cantab BC: Prophylactic inoculation against hay fever. Lancet 205(4580), 1572-1573 (1911).

2 Martínez-Gómez JM, Johansen P, Erdmann I, Senti G, Crameri R, Kündig TM:

Intralymphatic injections as a new administration route for allergen-specific immunotherapy. Int. Arch. Allergy Immunol. 150(1), 59-65 (2009).

3 Senti G, Johansen P, Kündig TM: Intralymphatic immunotherapy. Curr. Opin. Allergy Clin. Immunol. 9(6), 537-543 (2009).

4 Schmitz N, Dietmeier K, Bauer M et al.: Displaying Fel d1 on virus-like particles prevents reactogenicity despite greatly enhanced immunogenicity: a novel therapy for cat allergy. J. Exp. Med. 206(9), 1941-1955 (2009). 
5 Campbell JD, Buckland KF, McMillan SJ et al: : Peptide immunotherapy in allergic asthma generates IL-10-dependent immunological tolerance associated with linked epitope suppression. J. Exp. Med. 206(7), 1535-1547 (2009).

6 Focke M, Swoboda I, Marth K, Valenta R: Developments in allergen-specific immunotherapy: from allergen extracts to allergy vaccines bypassing allergen-specific immunoglobulin E and T cell reactivity. Clin. Exp. Allergy 40 (3), 385-397 (2010).

7 Novak N, Gros E, Bieber T, Allam JP: Human skin and oral mucosal dendritic cells as 'good guys' and 'bad guys' in allergic immune responses. Clin. Exp. Immunol. 161(1), 28-33 (2010).

8 Jutel M, Akdis M, Budak F et al.: IL-10 and TGF- $\beta$ cooperate in the regulatory $\mathrm{T}$ cell response to mucosal allergens in normal immunity and specific immunotherapy. Eur. J. Immunol. 33(5), 1205-1214 (2003).
9 Meiler F, Zumkehr J, Klunker S, Rückert B, Akdis CA, Akdis M: In vivo switch to IL-10-secreting T regulatory cells in high dose allergen exposure. J. Exp. Med. 205(12), 2887-2898 (2008).

10 Palomares O, Yaman G, Azkur AK, Akkoc T, Akdis M, Akdis CA: Role of Treg in immune regulation of allergic diseases. Eur. J. Immunol. 40(5), 1232-1240 (2010).

11 Klunker S, Chong MM, Mantel PY et al.: Transcription factors RUNX1 and RUNX3 in the induction and suppressive function of Foxp $3^{+}$inducible regulatory $\mathrm{T}$ cells. J. Exp. Med. 206(12), 2701-2715 (2009).

12 Cox L, Larenas-Linnemann D, Lockey RF, Passalacqua G: Speaking the same language: The World Allergy Organization Subcutaneous Immunotherapy Systemic Reaction Grading System. J. Allergy Clin. Immunol. 125(3), 569-574 (2010). 